\title{
Radical Surgical Excision and Use of Lateral Thoracic Flap for Intractable Axillary Hidradenitis Suppurativa
}

\author{
Wan-Lin Teo ${ }^{1,2}$, Yee-Siang Ong ${ }^{1}$, Bien-Keem Tan ${ }^{1}$ \\ ${ }^{1}$ Department of Plastic, Reconstructive and Aesthetic Surgery, Singapore General Hospital; ${ }^{2}$ National Skin Centre, Singapore
}

Current treatments for hidradenitis suppurativa (HS) include prolonged courses of antibiotics, retinoids, immunosuppressants, and biologics. Severe cases that are resistant to prolonged medical treatment pose a therapeutic challenge. We propose radical excision and lateral thoracic flap reconstruction as a treatment option for such cases. In our experience with two patients, good aesthetic and functional outcomes were achieved, with a high level of patient satisfaction. The availability of suitable flap coverage allows for wide resection of all of the hair-bearing skin, leading to a low incidence of residual disease and subsequent recurrence. Following excision of the affected tissue, the ideal reconstructive method in the axilla provides suitable coverage without unacceptable donor site morbidity and also avoids axillary contractures. A long lateral thoracic flap with delay has excellent coverage with minimal donor tissue sacrifice. With a suitable flap coverage option, the management paradigm of intractable HS should shift from prolonged medical treatment to allow decisive radical excision, which will improve the quality of life for patients.

Keywords Hidradenitis / Surgical flaps / Axilla
Correspondence: Bien-Keem Tan Department of Plastic, Reconstructive and Aesthetic Surgery, Singapore General Hospital, Outram Road, Singapore 169608

Tel: $+65-6321-4686$

Fax: +65-6225-9340

E-mail: bienkeem@singnet.com.sg

This paper was presented as an oral presentation at the Joint 15th Association of Southeast Asian Nations (ASEAN) Congress of Plastic Surgery and the 8th Asian Pacific Craniofacial Conference on July 15-17, 2010 in Kuching, Sarawak, Malaysia.

No potential conflict of interest relevant to this article was reported.

Received: 20 May 2012 • Revised: 3 Oct 2012 • Accepted: 17 Oct 2012

pISSN: 2234-6163 • elSSN: 2234-6171 • http://dx.doi.org/10.5999/aps.2012.39.6.663 • Arch Plast Surg 2012;39:663-666

\section{INTRODUCTION}

Hidradenitis suppurativa (HS) of the axilla is a chronic debilitating disorder of the apocrine glands. In severe cases, follicular occlusion and secondary apocrinitis lead to the formation of abscesses and fistulating sinus tracts, which are foul smelling, painful, and eventually cause scars [1]. Current treatments include prolonged courses of antibiotics, retinoids, immunosuppressants, and biologics. When there is no response to medical treatment and when activities of daily living are affected, these cases become intractable. We define HS as intractable when there is no response to medical therapy for at least 6 months. For such cases, we propose performing radical excision and flap coverage. We describe our experience with two patients who had intractable HS, necessitating radical excision and fasciocutaneous flap reconstruction.

\section{Surgical technique}

In patients with acute inflammation and abscesses, saucerisation and drainage are performed before planning flap coverage. A topical negative pressure dressing is applied in the interim period. Those who present with chronic discharging sinuses are candidates for excision and early definitive flap coverage during the same surgical sitting. Preoperative markings of the lateral 
thoracic flap are made with the patient in the supine position. The flap axis is centred over the posterior axillary line (Fig. 1). Doppler imaging is used to identify perforators in the vicinity, and to centre the flap over the loudest signal. With the shoulder abducted, two parallel skin incisions are made and extended to the lateral thoracic fascia.

The tip of the flap is undermined but not detached. The donor site is then closed in layers over the drains, with the overlying flap bipedicled like a handle. The tip is gradually divided over 2 weeks. When fully released, the flap is transposed to cover the defect, which must be clean.

\section{CASE}

\section{Case 1}

A 19-year-old male presented with recurrent axillary abscesses that had been present for the previous 2 years with severe scarring that impaired shoulder movement (Fig. 2). Prolonged medical treatment with oral antibiotics, retinoids, dapsone, and repeated incision and drainage were unsuccessful. He underwent excision followed by the application of topical negative pressure dressings. Bilateral lateral thoracic fasciocutaneous flaps were used for coverage. In view of the larger right axillary defect, a

\section{Fig. 1. Surgical technique}

(A) One week after delay, radical excision of the affected axillary tissue was performed. The ' $X$ ' marks the Doppler signal of the perforator supplying the base of the flap. (B) The delayed flap was raised and transposed onto the axillary defect. (C) Wound closure with drain in situ.
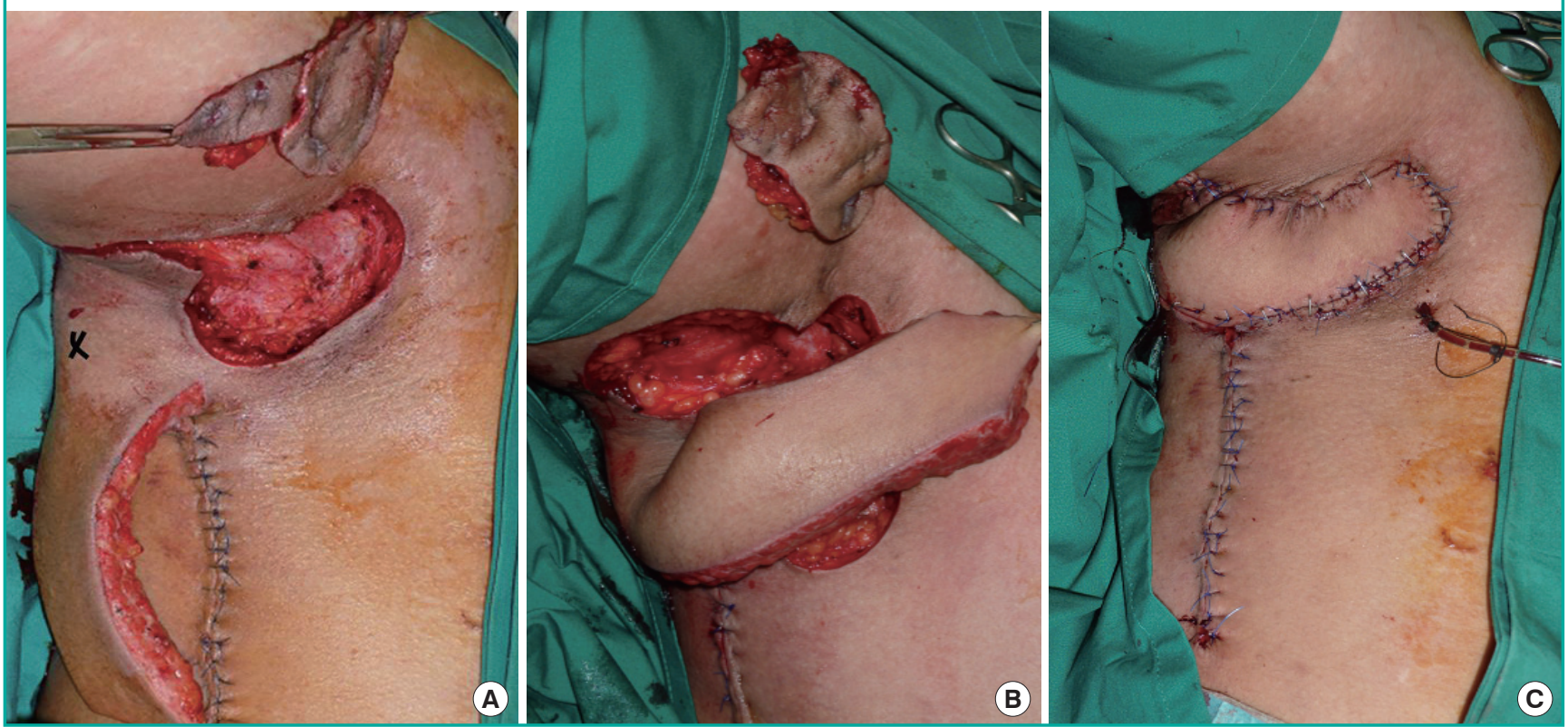

\section{Fig. 2. Case 1}

(A) Intractable HS presenting with multiple nodules and with sinuses that had foul smelling pus. The range of motion at both axillae was impaired, the right side was worse than the left. (B) Three years postoperatively, the patient regained full range of motion with good wound healing and preservation of aesthetics.
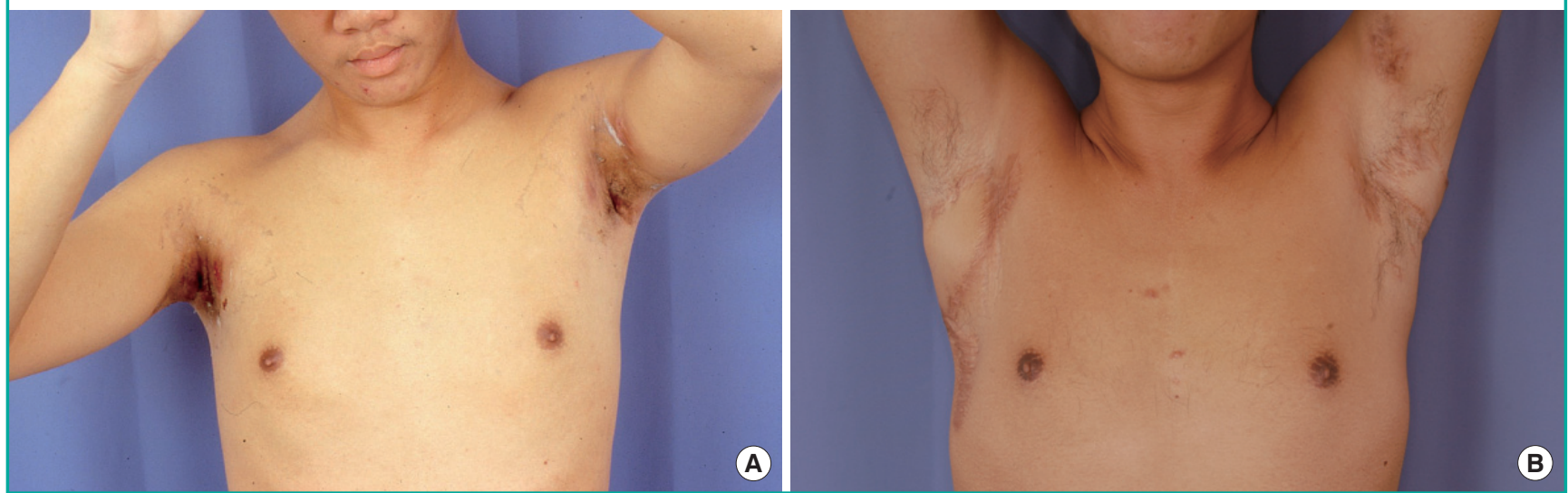


\section{Fig. 3. Case 2}

(A) The patient had severe scarring from bilateral axillary hidradenitis suppurativa, which had affected his delivery job. He also had limited range of motion at the shoulders. (B) Six months postoperatively, the patient showed good wound healing and improved shoulder excursion.

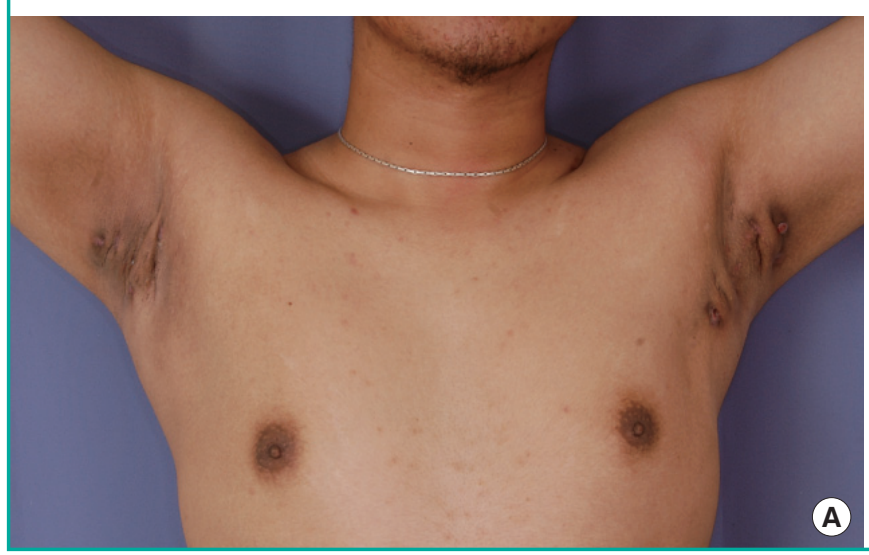

(A)

decision was made to delay the flap. The left flap was applied without delay because the defect was smaller. The drains were removed on postoperative day 2 , and the patient was discharged on oral antibiotics. Full range of arm movement was regained on the subsequent follow-up. He subsequently underwent minor scar revision 2 years later.

\section{Case 2}

A 21-year-old male who was a heavy smoker presented with bilateral axillary HS that had existed for 3 years and had affected his job doing delivery work (Fig. 3). In view of his heavy smoking, the delay technique was employedo safeguard the tip of the flap. He underwent excision and flap reconstruction in stages. Drains were removed on postoperative day 2 and he was discharged without incident on oral antibiotics on postoperative day 3 . Six months postoperatively, all of the wounds were healed and he had full range of shoulder movement. In both of the cases, the flaps survived completely and good aesthetic and functional outcomes were achieved.

\section{DISCUSSION}

Surgery is indicated for severe HS [2]. The affected tissue should be excised with wide margins and special efforts should be made to eliminate all deeply burrowed glands. The resultant defect, if allowed to heal secondarily, may result in contractures and shoulder stiffening. To avoid this, primary healing using direct closure, split-skin grafting, or local flap application is recommended. Direct closure is associated with a high incidence of recurrence, presumably because of limited resection, while skin grafting poses problems of graft take, repeated dressings, cosmesis, and scarring. A flap is a good option because it provides reliable soft

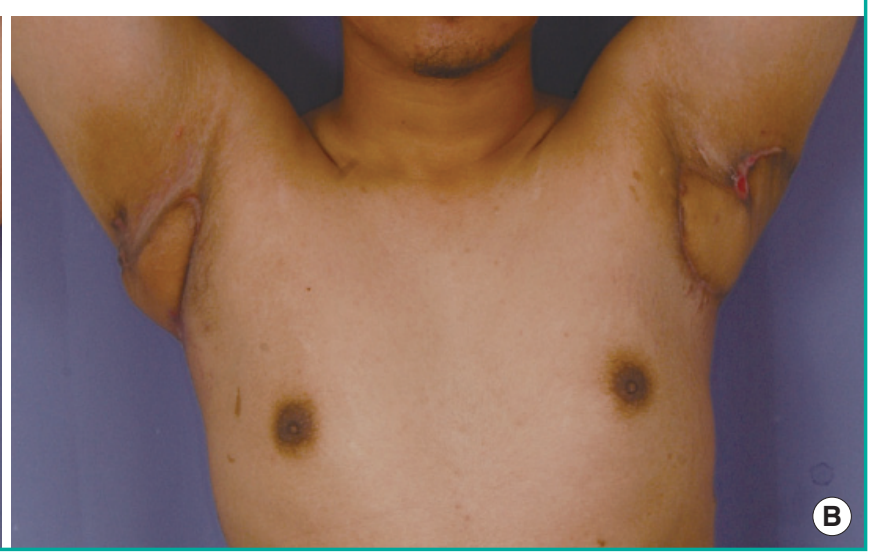

tissue coverage while avoiding axillary contractures. The skin of the axilla and lateral thoracic wall is supplied by perforators originating from three vessels, namely the lateral thoracic, thoracodorsal, and accessory lateral thoracic arteries. These three vessels arise from the axillary artery. The lateral thoracic flap is designed based on blood supply from these perforators; it is robust, with dimensions reaching $30 \times 15 \mathrm{~cm}$. The delay technique was employed to boost the blood supply of the flap tip to ensure its survival. The first stage allowed thorough excision, debridement, and topical negative pressure therapy of the contaminated wound, and also allowed the lateral thoracic flap to be partially raised. The second stage involved transposition of the flap onto the healthy granulating wound bed of the axillary defect.

The delay phenomenon maximizes the survival of a flap by improving its circulation. Collateral blood supply is interrupted by partially raising the flap. When this occurs, "choke" vessels, which are connectors between adjacent vascular territories, open up in response to ischemia, hence directing blood flow into the distal portions of the flap. The blood vessels in the flap also increase in caliber, thereby improving blood flow. Additionally, endothelial progenitor cells are recruited for angiogenesis [3].

Another perspective is that the lateral thoracic flap is a randompatterned flap because delay would have been unnecessary if it were a perforator-based flap. Nevertheless, delay was employed to safeguard the tip of the flap because of infection and the patient's history of heavy smoking.

Different types of advancement and transposition flaps for reconstruction after excision of axillary hidradenitis have been described $[4,5]$. Available flap options include islanded flaps such as the thoracodorsal artery perforator flap [4] and the V-Y advancement flap [5], which rely on underlying perforators for circulation. The Limberg flap is a rhomboid-shaped transposi- 
tion flap, which has been described for axillary coverage. The lateral thoracic transposition flap is similar, except that it offers a longer flap and a more robust tip with the delay technique $[6,7]$. In intractable axillary hidradenitis suppurativa, the availability of suitable flap coverage allows for wide resection of all of the hair-bearing skin, leading to a low incidence of residual disease and subsequent recurrence. This also provides an important perspective for physicians seeking an effective, permanent treatment for HS rather than long-term medical therapy, which itself is associated with significant morbidity.

\section{REFERENCES}

1. Shelley WB, Cahn MM. The pathogenesis of hidradenitis suppurativa in man; experimental and histologic observations. AMA Arch Derm 1955;72:562-5.

2. Soldin MG, Tulley P, Kaplan H, et al. Chronic axillary hi- dradenitis: the efficacy of wide excision and flap coverage. $\mathrm{Br}$ J Plast Surg 2000;53:434-6.

3. Ghali S, Butler PE, Tepper OM, et al. Vascular delay revisited. Plast Reconstr Surg 2007;119:1735-44.

4. Rehman N, Kannan RY, Hassan S, et al. Thoracodorsal artery perforator (TAP) type I V-Y advancement flap in axillary hidradenitis suppurativa. Br J Plast Surg 2005;58:441-4.

5. Schwabegger AH, Bodner G, Ninkovic M, et al. Thoracodorsal artery perforator (TAP) flap: report of our experience and review of the literature. Br J Plast Surg 2002;55:390-5.

6. O’Brien J, Wysocki J, Anastasi G. Limberg flap coverage for axillary defects resulting from excision of hidradenitis suppurativa. Plast Reconstr Surg 1976;58:354-8.

7. Varkarakis G, Daniels J, Coker K, et al. Treatment of axillary hidradenitis with transposition flaps: a 6-year experience. Ann Plast Surg 2010;64:592-4. 